\title{
in vitro MYOGENIC DIFFERENTIATION OF BOVINE MULTIPOTENT MESENCHYMAL STEM CELLS TAKEN FROM BONE MARROW AND ADIPOSE TISSUE
}

\author{
I.A. Rogov ${ }^{1}$, I.M. Volkova, ${ }^{1,2}$ K.V. Kuleshov ${ }^{2}$, I.P. Savchenkova ${ }^{2}$ \\ ${ }^{1}$ Moscow National University of Food Production, Moscow 109316, Russia \\ ${ }^{2}$ Ya.R. Kovalenko All-Russia Research and Development Institute of Experimental Veterinary, RAAS, Moscow 109428, Russia \\ e-mail: akoulinairina@mail.ru,s-ip@mail.ru,konstantinkul@gmail.com
}

Received September 28, 2012

Sum mary

Bovine bone marrow (BM) and adipose tissue (AT) multipotent mesenchymal stem cells (MMSC) are believed to be a perspective material for the creation of new cellular systems and products for food biotechnology. We demonstrated the possibility of directed differentiation of BM and AT MMSC in those of muscle tissue by culturing them in the medium, containing inductors of myogenesis (5-azacytidine, 5-aza-2'-deoxycytidine and retinoic acid). The comparative analysis of differentiation efficiency of these cells in the direction of myogenesis revealed morphological changes in BM MMSC on the 15-21st day, and in AT MMSC - on the 20-25th day of culturing in the medium with different inductors. The analysis of MYOD1 and MYOG gene expression in RT-PCR detected essential distinctions in the potency of these cells to differentiate into muscle tissue cells in vitro, and also showed that MMSC, isolated from BM, are more perspective source for muscle tissue engineering in vitro.

Keywords: multipotent mesenchymal stem cells, bone marrow, adipose tissue, bovine, differentiation, myogenesis, in vitro, inductors, gene expression.

Today, many countries (the Netherlands, USA, Japan, Australia, etc.) perform the experiments on production of in vitro cultured meat (1-2) that may be an alternative source of animal protein. A group of Russian researchers headed by I.A.Rogov developed the unique method of accumulation of muscle cells (3). These works were continued by experiments on multipotent mesenchymal stem cells (MMSCs) derived from bone marrow (BM) and adipose tissue (AT) of cattle, that can be a possible source of accumulated cellular biomass.

MMSCs of farm animals are in focus of scientific interest due to their main characteristics and features. First, these cells have the ability to self-renewal in vitro not being involved in aneuploidy, genetic instability, and malignant transformation; cultured MMSCs proliferate for a long time and form stable diploid cell lines. Secondly, when MMSCs are induced to differentiate in vitro, they develop to the cells of other tissues. Another advantage of cultured MMSCs is their ability to fill a three-dimensional scaffold matrix resulting in a structural simulant of various tissues grown in vitro (4). Therefore, MMSCs can be the unique material for creation of new cellular systems and bioengineered agricultural products including food. This target is fairly attainable owing to high availability of a biological material (BM and AT) used as a source of the cells with properties and attributes of MMSCs.

There are many reports about such cells isolated from BM and AT of a human (5-6), rodents (7), and agricultural animals (8$12)$; when induced in vitro, they develop to the cells of bone tissue, cartilage, and adipose tissue. It has been also shown that MMSCs of a human (6), rodents (13), rabbits (14), cattle, and pigs (9) under certain conditions in vitro may form the cells of muscle tissue. These facts underlie a new promising direction of scientific research in different areas - medicine, veterinary, biology, and biotechnology.

Earlier, the authors have isolated from BM and AT of cattle and described the populations of cells phenotypically related to MMSCs. When both these cell populations were cultured in an induction medium in vitro, they developed the cells of bone and adipose tissue (12), but the potency of MMSCs for targeted differentiation in muscle cells in vitro wasn't studied.

In connection with these facts, the presented work was aimed at comparing the ability of multipotent mesenchymal stem cells of different origin to targeted differentiation in vitro to the muscle cells at the level of expression of myogenic marker genes.

Technique. MMSCs derived from BM and AT of cattle were induced for differentiation in vitro to muscle cells according to the previously described procedures $(9,13,14)$. At the fifth passage, the cells were planted in 6 -well plates $\left(5 \frac{1}{2} 10^{3}-61 / 210^{3}\right.$ per well; well diameter $35 \mathrm{~mm}$ ) and after 24 hours they were subject to different induction media. The base medium for culturing was a lowglucose (1 g/l) DMEM (DMEM-LG) supplemented with 10\% fetal bovine serum (FBS) ("HyClone", USA) and antibiotics. The final concentration of streptomycin was $100 \mathrm{ug} / \mathrm{ml}$, penicillin - $100 \mathrm{units} / \mathrm{ml}$.

Composition of the induction media: I - DMEM-LG, 10\% FBS, retinoic acid (12 uM); II - DMEM-LG, 15\% FBS, 5azacytidine (10 uM), hydrocortisone (50 uM); III - DMEM-LG, 15\% FBS, 5-aza-2'-deoxycytidine (0,3 uM), hydrocortisone (50 uM).

Culturing in the induction media supplemented with 5-aza-cytidine and 5-aza-2'-deoxycytidine ("Sigma", USA) was performed for 24 hours, in the medium with retinoic acid ("Sigma", USA) - 4 days (the induction medium was discarded daily). Then the induction medium was replaced with the base medium (free from the inducer) and then the cells were cultured in $\mathrm{CO}_{2}$-incubator at $5 \% \mathrm{CO}_{2}$ and $37^{\circ} \mathrm{C}$

To assess the potential of MMSCs for differentiation to muscle cells in vitro, all the samples were divided to six experimental groups: BM MMSCs - groups 1, 2, and 3, AT MMSCs - groups 4, 5, and 6. The groups 1 and 2 were treated with I induction medium, groups 2 and 5 - II induction medium, groups 3 and 6 - III induction medium. The effect of the inducers was studied as dynamics recorded on the $21^{\text {st }}, 28^{\text {th }}$, and $33^{\text {rd }}$ days of differentiation. Control cells were cultured in medium without inducers.

Morphological changes in MMSCs during differentiation were evaluated visually in both native and stained (RomanovskyGiemza) specimens using an inverted phase-contrast microscope ("Carl Zeiss", Germany) and the program Ax-ioVision Rel. v. 4.8. Intracellular accumulation of neutral fats was revealed by staining lipid inclusions with Oil Red O ("Bio Optica Milano SPA", Italy).

The fact of myogenic differentiation was confirmed by evaluation of the expression of mRNA - the product of genes MYODI and MYOG known as myogenic markers (using reverse transcription followed by polymerase chain reaction - RT-PCR) relative to the level of a constantly expressed $G A P D H$ gene of glyceraldehyde-3-phosphate dehydrogenase. For this purpose, on the 
$21^{\text {st }}$ day of differentiation, a part of the cells was used to derive the total mRNA. Total mRNA was isolated from the cells using Mini RNA Isolation II Kit ("Zymo Research", USA). Elution was conducted in 30 ul water (DNAase RNAase free). To perform the reverse transcription, $10 \mathrm{ul}$ of the prepared RNA solution was added with 1 ul Random Hexamer Primer ("Fermentas", Lithuania), incubated at $70{ }^{\circ} \mathrm{C}$ for $5 \mathrm{~min}$, then the reaction mixture was placed on ice for $2 \mathrm{~min}$. Then, $11 \mathrm{ul}$ of this solution was added with $8,5 \mathrm{ul}$ of the reaction mixture for reverse transcription containing 4 ul 5× reaction buffer, $1 \mathrm{mM}$ of each dNTP ( ${ }^{1}$ R0191, "Fermentas", Lithuania) and 40 units M-MuLV reverse transcriptase ( ${ }^{1}$ EP0351, "Fermentas", Lithuania) This reaction mixture was incubated for 10 min at 25 ${ }^{\circ} \mathrm{C}$, then 60 minutes at $42{ }^{\circ} \mathrm{C}$. Immediately after the reverse transcription, the reaction mixture was diluted with TE-buffer up to twice volume.

During PCR for each of the test targets it was prepared individual reaction volume of 25 ul containing: 5 ul cDNA matrix (5$20 \mathrm{ng}$ ), $1 \times$ reaction buffer (PCR-mix-2-FRT, “Amplisen”, Russia), 1 unit Taq-F polymerase (“Amplisen”, Russia), 200 uM of each dNTP (№ R0191, "Fermentas”, Lithuania), forward and reverse primers (240 nM each one) ("Litech”, Russia). The reaction was performed on thermocycler Tertsik (Russia). Regime of amplification: $95{ }^{\circ} \mathrm{C}, 15 \mathrm{~min}$ (preliminary denaturation and activation of TaqF polymerase), then 30 cycles $\left(95^{\circ} \mathrm{C}, 20 \mathrm{sec}\right) ; 60^{\circ} \mathrm{C}, 20 \mathrm{sec} ; 72^{\circ} \mathrm{C}, 20 \mathrm{sec}$.

Electrophoresis of DNA fragments was performed in $2 \%$ agarose gel in Tris-acetate buffer (TAE). DNA was visualized by staining the gel with ethidium bromide solution in TAE $(5 \mathrm{ug} / \mathrm{ml})$ for $10-20$ minutes. The gels were viewed in ultra-violet light $\lambda=254$ $\mathrm{nm}$ and documented. Fragment size was determined using molecular weight marker ("Promega", USA).

Results. Since the work was performed not using the dyes that reveal cellular differentiation (eg., monoclonal antibodies (MAbs) against antigens (AG) of cattle), this fact was confirmed by visual observations of morphological changes in the cells treated with the inducers of myogenesis, along with RT-PCR evaluation of mRNA expression on myogenic marker genes. The used primers complementary to transcripts of the studied genes are shown in the table.

Forward (F) and reverse (R) primers used for amplification of the reversed transcripts for genes MYOD1 and MYOG (markers of myogenesis) and constantly expressing gene GAPDH (glyceraldehyde-3-phosphate dehydrogenase)

\begin{tabular}{c|c|l}
\hline Target & Oligonucleotide & \multicolumn{1}{c}{ Direction $5^{\prime} \rightarrow 3^{\prime}$} \\
\hline GAPDH & Gab_F & TCA-TTG-ACC-TTC-ACT-ACA-TGG-TCT-A \\
& Gab_R & AAG-ATG-GTG-ATG-GCC-TTT-CCA-TTG \\
MYOD1 & MY_F & GCT-CCA-GAA-CAG-CAG-CAA-GT \\
& MY_R & TCG-AAA-CAC-GGG-TCA-TCA-TA \\
MYOG & MG_F & CAG-TGA-ATG-CAG-CTC-CCA-TA \\
& MG_R & CGA-CAT-CCT-CCA-CTG-TGA-TG \\
\hline
\end{tabular}

The authors studied the effects of three induction media containing different agents - 5-azacytidine, 5-aza-2'-deoxycitidine (cytosine analogues with demethylating activity), and retinoic acid, which are known from the literature as inducers of in vitro formation of muscle cells $(9,13,14)$.

In the experimental groups $1,2,3-$ on the $5^{\text {th }}$ day, and in groups $4,5,6-$ on the $7^{\text {th }}$ day of culturing with the induction media I, II and III, it was observed the increase in cell size. The cells were stretching in one direction and merging; on the days $15^{\text {th }}-21^{\text {st }}$ (groups $1,2,3$ ) or $20^{\text {th }}-25^{\text {th }}$ (groups $4,5,6$ ), there were formed dense myotube-like structures each containing 3-10 nuclei.

Staining with Romanovsky-Giemsa dye showed the central position of the nuclei peculiar to the early stage of formation of myosymplasts. About $40 \%$ cells had visually detected morphological changes: they were getting rounded, compacted, elongated and multinuclear (Figure 1, A). Some of these cells became large with visible striations of the cytoplasm. (Fig. 1, B). Along with the formation of cell clusters, there also appeared lipid vesicles revealed by Oil Red $\mathrm{O}$ dye. The number of stained lipid vacuoles in the experimental groups 4,5 , and 6 was significantly greater than in groups 1,2 , and 3 . Control cells (cultured without the inducer) didn't show any morphological changes.

A

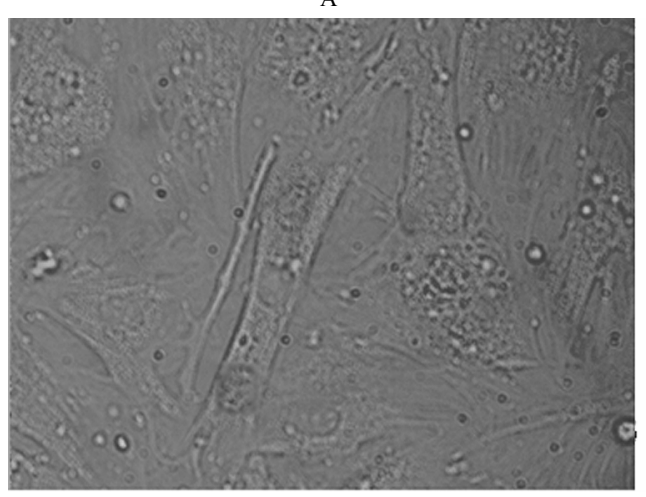

Fig. 1. Target differentiation of multipotent mesenchymal stem cells isolated from bone marrow (A) and adipose tissue (B) of cattle toward the cells of muscle tissue in vitro during a culturing in myogenic medium: $\mathrm{A}-$ the $28^{\text {th }}$ day (inducer -5 -azacytidine), native preparation; $\mathrm{B}-$ the $33^{\text {rd }}$ day (inducer - retinoic acid); stained with Romanovsky-Giemsa, magnification $\times 63$ (object-glass), $\times 10$ (eyeglass).
B

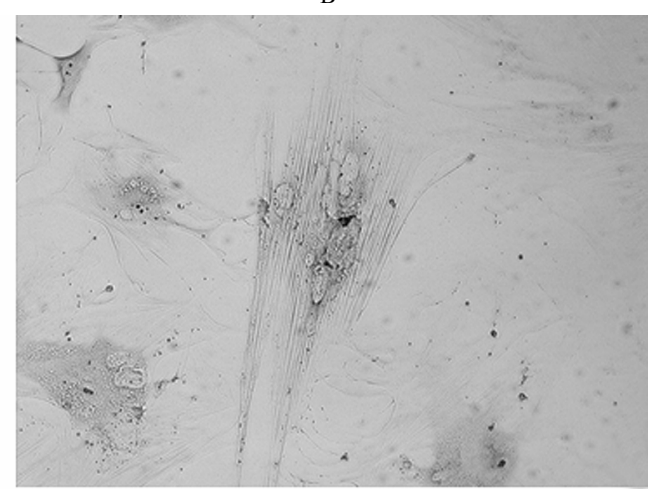

Studying the effects of various inducers, it was found that all of them stimulated MMSCs to transform in vitro to the cells of muscle tissue, though in the groups treated with II and III induction media, it was observed degeneration and death of about 5-10\% cells, while retinoic acid didn't show such a toxic effect.

Myogenesis is known to be regulated by a family of transcription factors MRF (myogenic regulatory factors) including MYOD1, MYF5, MYF6 and MYOG. During embryogenesis, MYOD1 and MYF5 are involved in establishing of the skeletal muscle line, while MYOG is enabled in terminal differentiation. MYOD1 is one of the early markers that evidences the initiation of myogenic differentiation of MMSCs; the expression of MYOD1 is characteristic to the phase of formation of myoblasts (15).

Visually detected morphological changes in MMSCs cultured in vitro were confirmed at the molecular level (Fig. 2). 

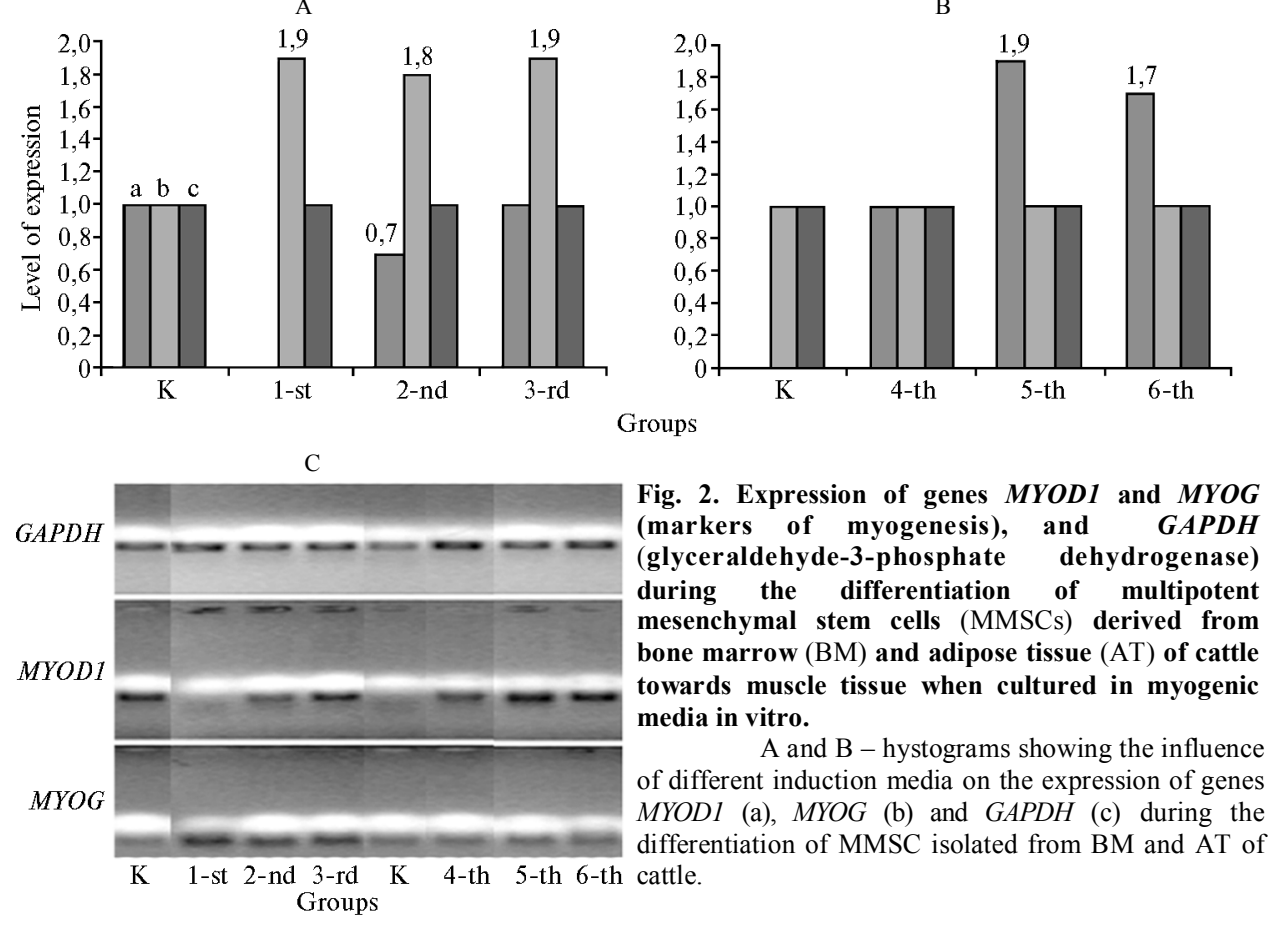

$\mathrm{C}$ - electrophoregram showing RT-PCR amplification of the products of genes MYOD1, MYOG, and GAPDH; $\mathrm{K}$ - culture of cells without inducers (control). The inducers of myogenesis - retinoic acid, 5-azacytidine, and 5azadeoxycitidine applied on, respectively, the $1^{\text {st }}, 2^{\text {nd }}$, and $3^{\text {rd }}$ experimental groups of BM-derived MMSCs, and the $4^{\text {th }}, 5^{\text {th }}$, and $6^{\text {th }}-$ AT-derived MMSCs.

During differentiation of MMSCs in the all six experimental groups, the product of $M Y O D 1$ gene expression was detected on the $21^{\text {st }}$ day in BM-derived MMSCs (experimental groups 2, 3, and BM-derived control). The expression of MYOD1 was also observed in AT-derived MMSCs (experimental groups 4, 5, and 6), while in AT-derived control it wasn't found. The histogram (Fig. 2) shows the level of expression of MYOD1 gene: in groups 5 and 6 it almost twice exceeded that in other groups. The mRNA of $M Y O G$ gene was detected in all six groups, but in groups 1,2 , and 3, the amount of this product was almost twice higher than in control samples and groups 4,5 , and 6 .

Along with experimental groups, the product of MYOD1 gene was also detected in BM-derived control MMSCs cultured in medium without the inducer, while in AT-derived MMSCs it was present only in the cells cultured in myogenic media. The presence of $M Y O D 1$ and $M Y O G$ gene products in the control samples can be explained by spontaneous myogenic differentiation. Probably, the absence of $M Y O D 1$ gene product in the experimental group 1 was associated with completion of the early stage of myogenesis and the formation of terminally differentiated myocytes. As it can be seen from the histogram (Fig. 2), BM-derived MMSCs showed a high level of $M Y O G$ gene expression and no expression of MYOD1 gene. On the contrary, AT-derived MMSCs showed a high level of MYOD1 gene expression along with the expression of $M Y O G$ gene. These data allow concluding that BM-derived MMSCs of cattle develop faster myogenic differentiation than AT-derived ones.

These findings are consistent with other published data $(9,13)$, but differ from results obtained by Z. Su et al. (14).

Thus, multipotent mesenchymal stem cell (MMSCs) derived from bone marrow (BM) and adipose tissue (AT) of cattle when cultured in media containing the inducers 5-azacytidine, 5-aza-2'-deoxycytidine, and retinoic acid, have demonstrated differentiation to muscle cells in vitro. In the authors' experiments on cultured MMSCs with retinoic acid added as the inducer of myogenic differentiation, its optimum concentration was established at $12 \mathrm{uM}$, while $50 \mathrm{uM}$ was found to be pernicious. The obtained results indicate that, despite the phenotypic similarity in populations of BM- and AT-derived MMSCs, these cells have different potencies to myogenesis. Comparative analysis of the level of expression of myogenic marker genes in the studied MMSCs showed that BMderived MMSCs have better capability for myogenic differentiation in vitro.

\section{REFERENCES}

1. Edelman P.D., McFarland D.C., Mironov V.A., and Matheny J.G., In vitro-Cultured Meat Production, Tissue engineering, 2005, vol. 11, no. 5/6, pp. 659-662.

2. Haagsman H.P., Hellingwerf K.J., and Roelen B.A.J., Production of Animal Proteins by Cell Systems, Utrecht: Faculty of Veterinary Medicine, October 2009.

3. Rogov I.A., Valikhov A.F., Demin N.Ya., Krokha N.G., Lisitsyn A.B., Semenov G.V., Titov E.I., Tutelyan V.A., Rogov S.I., and Ernst L.K., Method for Producing Tissue Engineered Meat for Consumption, Patent № 2314719 RF. MPK 7 C12N 5/06, A 23 L 1/31/; applicant and patent owner Moscow State University of Applied Biotechnology, № 2006119540. Appl. 06.06.2006, Publ. 20.01.2008, Bull. № 2.

4. Savchenkova I.P. and Gulyukin M.I., Prospects of Using Stem Cells in Veterinary, Veterinariya, 2011, vol. 7, pp. 3-5.

5. Teplyashin A.S., Korzhikova S.V., Sharivullina S.Z., Chupikova N.I., Rostovskaya M.S., and Savchenkova I.P., Characterization of Mesenchymal Stem Cells Isolated from Human Bone Marrow and Adipose Tissue, Tsitologiya, 2005, vol. 47, no. 2, pp. 130-135.

6. Zuk P.A., Zhu M., Ashjian P., et al., Human Adipose Tissue is a Source of Multipotent Stem Cells, Mol. Biol. Cell, 2002, vol. 13, no. 1, pp. 4279-4295.

7. Lennon D.P. and Caplan A.I., Isolation of Rat Marrow-Derived Mesenchymal Stem Cells, Exp. Hematol., 2006, vol. 34, no. 11, pp. 1606-1607.

8. Bosnakovski D., Mizuno M., Kim G., et al., Isolation and Multilineage Differentiation of Bovine Bone Marrow Mesenchymal Stem Cells, Cell Tissue Res., 2005, vol. 319 , no. 2 , pp. $243-253$.

9. Colleoni S., Donofrio G., Lagutina I., et al., Establishment, Differentiation, Electroporation, Viral Transduction, and Nuclear Transfer of Bovine and Porcine Mesenchymal Stem Cells, Cloning and Stem Cells, 2005, vol. 7, no. 3, pp. 154-166.

10. Rentsch C., Hess R., Rentsch B., et al., Ovine Bone Marrow Mesenchymal Stem Cells: Isolation and Characterization of the Cells and Their Osteogenic Differentiation Potential on Embroidered and Surface-Modified Polycaprolactone-co-Lactide Scaffolds, In vitro Cell. Develop. Biol. - Animal, 2010, vol. 46, no. 7, pp. 624-634.

11. Smith R.K., Korda M., Blunn G.W., and Goodship A.E., Isolation and Implantation of Autologous Equine Mesenchymal Stem Cells from Bone Marrow into the 
Superficial Digital Flexor Tendon as a Potential Novel Treatment, Equine Vet. J., 2003, vol. 35, no. 1, pp. 99-102.

12. Volkova I.M., Viktorova E.V., Savchenkova I.P., and Gulyukin M.I., Characteristic of Mesenchymal Stem Cells Isolated from Bone Marrow and Fatty Tissue of Cattle, S.-kh. biol., 2012, vol. 2, pp. 32-38.

13. Wakitani S., Saito T., and Caplan A.I., Myogenic Cells Derived from Rat Bone Marrow Mesenchymal Stem Cells Exposed to 5-Azacytidine, Muscle \& Nerve, 1995, vol. 18, pp. 1417-1426.

14. Su Z., Li Y., Zhao X., and Zhang M., All-Trans Retinoic Acid Promotes Smooth Muscle Cell Differentiation of Rabbit Bone Marrow-Derived Mesenchymal Stem Cells, Journal of Zhejiang University-SCIENCE B (Biomed. Biotechnol.), 2010, vol. 11, no. 7, pp. 486-496.

15. Strazzullo M., Rossetti C., Fusco G., et al., Genomic Characterization and Chromosomal Mapping of 5 River Buffalo Skeletal Muscle Differentiation Master Genes, Cytogen. Genome Res., 2010, vol. 128, pp. 221-227. 